Published in final edited form as:

Exp Eye Res. 2013 November ; 116: . doi:10.1016/j.exer.2013.08.017.

\title{
Autophagy and mitophagy participate in ocular lens organelle degradation
}

\author{
M. Joseph Costello ${ }^{\# 1,2}$, Lisa A. Brennan ${ }^{3}$, Subharsee Basu ${ }^{4}$, Daniel Chauss ${ }^{3}$, Ashik \\ Mohamed $^{5}$, Kurt O. Gilliland ${ }^{2}$, Sönke Johnsen ${ }^{6}$, Sue Menko ${ }^{4}$, and Marc Kantorow ${ }^{\# 1,3}$ \\ ${ }^{2}$ Department of Cell Biology and Physiology, University of North Carolina, Chapel Hill, NC \\ ${ }^{3}$ Department of Biomedical Science, Florida Atlantic University, Boca Raton, FL. \\ ${ }^{4}$ Department of Pathology, Anatomy and Cell Biology, Thomas Jefferson University \\ ${ }^{5}$ Prof. Brien Holden Eye Research Centre, L V Prasad Eye Institute, Hyderabad, India \\ ${ }^{6}$ Biology Department, Duke University, Durham, NC. \\ \# These authors contributed equally to this work.
}

\begin{abstract}
The eye lens consists of a layer of epithelial cells that overlay a series of differentiating fiber cells that upon maturation lose their mitochondria, nuclei and other organelles. Lens transparency relies on the metabolic function of mitochondria contained in the lens epithelial cells and in the immature fiber cells and the programmed degradation of mitochondria and other organelles occurring upon lens fiber cell maturation. Loss of lens mitochondrial function in the epithelium or failure to degrade mitochondria and other organelles in lens fiber cells results in lens cataract formation. To date, the mechanisms that govern the maintenance of mitochondria in the lens and the degradation of mitochondria during programmed lens fiber cell maturation have not been fully elucidated. Here, we demonstrate using electron microscopy and dual-label confocal imaging the presence of autophagic vesicles containing mitochondria in lens epithelial cells, immature lens fiber cells and during early stages of lens fiber cell differentiation. We also show that mitophagy is induced in primary lens epithelial cells upon serum starvation. These data provide evidence that autophagy occurs throughout the lens and that mitophagy functions in the lens to remove damaged mitochondria from the lens epithelium and to degrade mitochondria in the differentiating lens fiber cells for lens development. The results provide a novel mechanism for how mitochondria are maintained to preserve lens metabolic function and how mitochondria are degraded upon lens fiber cell maturation.
\end{abstract}

\section{Keywords}

Lens; Differentiation; Autophagy; Mitophagy; Cataract; Electron Microscopy

\footnotetext{
(C) 2013 Elsevier Ltd. All rights reserved.

${ }^{1}$ Correspondence to both authors.
}

Publisher's Disclaimer: This is a PDF file of an unedited manuscript that has been accepted for publication. As a service to our customers we are providing this early version of the manuscript. The manuscript will undergo copyediting, typesetting, and review of the resulting proof before it is published in its final citable form. Please note that during the production process errors may be discovered which could affect the content, and all legal disclaimers that apply to the journal pertain. 


\section{Introduction}

Organelle degradation is a major feature of the programmed differentiation events that lead to the formation of the mature vertebrate eye lens. The lens is a transparent, avascular tissue whose function is to focus light onto the retina (Bassnett et al., 2011).The adult lens is encapsulated and consists of a monolayer of cuboidal epithelial cells on the anterior surface that overlie layers of fiber cells formed by differentiation of epithelial cells at the lens equator that elongate toward the poles of the lens to form the lens fiber cells. Maturation of the lens fiber cells involves the loss of their mitochondria, nuclei and other organelles. The cells of the lens and their contents must remain viable throughout the entire lifespan. Damage to these cells and their contents results in cataract formation that is a opacity of the eye lens and a leading cause of vision loss. The lens epithelium contains important metabolic enzymes, ion-transport proteins and active energy producing mitochondria in the lens (Brown and Bron, 1996; Bloemendal, 1981). The lens epithelium requires mitochondrial activity to maintain lens homeostasis and damage to the lens epithelium or its mitochondria and many enzyme systems, leads to loss of lens transparency and cataract formation (Brennan and Kantorow, 2009; Delamere and Tamiya, 2009).

Immature lens fibers require mitochondria to generate energy for protein synthesis, cellular remodeling and other essential processes. During the transition to mature lens fibers, the lens fiber cells lose their mitochondria (Bassnett, 1992), nuclei (Bassnett, 1992), Golgi apparatus, and endoplasmic reticulum (Bassnett, 1997). Initiation of mitochondrial degradation during this process precedes nuclear degradation (Bassnett, 1992) suggesting that mitochondrial degradation is a key step in lens fiber cell maturation. Proteasomal and DNAse II -mediated degradation pathways have been demonstrated to be associated with organelle loss in the lens (Zandy and Bassnett, 2007; Girao et al., 2005; Bassnett, 2009; De Maria and Bassnett, 2009). The specific mechanisms accounting for the degradation of mitochondria and other organelles during lens fiber cell maturation are not known.

Crucial to the maturation of lens is the formation of an organelle free zone (OFZ) providing minimal scattering from cellular components along the light path (Rabl, 1899; Bloemendal, 1981; Brown and Bron, 1996; De Maria and Bassnett, 2009; Bassnett et al., 2011).

Incomplete removal of mitochondria has been shown to produce light scattering in cortical fiber cells in both mouse and chick models (Pendergrass et al., 2005). Retention of nuclear fragments in these outer lens fiber cells in a variety of species contributes to light scattering and cortical cataract in humans (Pendergrass et al., 2005; Pendergrass et al., 2011). Cellular debris remaining from organelle breakdown has been hypothesized as the source of globular particles, called multilamellar bodies, that are subcellular lens structures consisting of a cytoplasmic core surrounded by multiple layers of lipid bilayers (Gilliland et al., 2001). Multilamellar bodies have been shown to be likely sources of light scattering in human agerelated nuclear cataracts, the most common form of human cataract causing visual impairment (Gilliland et al., 2001; Gilliland et al., 2004; Costello et al., 2007). Deletion of the key mitochondrial repair enzyme, methionine sulfoxide reductase A, results in cataract formation in mice treated with hyperbaric oxygen (Brennan et al., 2009; Kantorow et al., 2012) and failure to repair and maintain the functions of mitochondrial enzymes results in lens epithelial cell death (Brennan et al., 2012). Thus, understanding how damaged mitochondria are degraded to maintain a healthy population of mitochondria in the lens epithelium and how mitochondria and other organelles are specifically degraded during programmed lens cell fiber differentiation is critical for our understanding of the cellular mechanisms that govern lens homeostasis and the development of the lens.

One major mechanism that accounts for the specific degradation of mitochondria and other organelles in many tissues is the process of autophagy, whereby cellular organelles and 
other components are specifically sequestered by double-layered membranes called autophagosomes. Selective degradation of mitochondria using the autophagy machinery is termed mitophagy (Youle and Narendra, 2011; Youle and van der Bliek, 2012; Itakura et al., 2012). Mitophagy is specific to for the autophagic degradation of damaged mitochondria and mitochondria that are targeted for elimination during organelle loss that occurs upon cellular maturation of erythrocytes (Youle and Narendra, 2011; Youle and van der Bliek, 2012; Itakura et al., 2012, Gronowicz et al., 1984, Mortensen et al, 2010). Autophagosomes containing mitochondria or other organelles and/or cellular material are ultimately delivered to and fused with lysosomes to form single membrane structures called the autophagolysosome where the contents are degraded (Levine and Klionsky, 2004). Autophagosomes are characterized by the presence of microtubule-associated protein 1 light chain 3B (LC3B) in their membranes. LC3B is a commonly used marker for the presence of autophagic vesicles (Klionsky et al., 2008).

Two reports have suggested that organelle degradation upon maturation of lens cells is independent of autophagy (Matsui et al, 2006; Morishita et al., 2013). One study was based on a knockout mouse deleted for the autophagy-related protein 5 (Atg5)-dependent initiation pathway (Matsui et al., 2006) and the second study examined ATG5- and PIK3C3/VSP34lens-targeted knockout mice (Morishita et al., 2013). Neither study rules out a role for autophagy in lens organelle degradation or formation of the organelle-free zone since these pathways are likely to be redundant and, importantly, autophagy occurs independently of ATG5 (Nishida et al., 2009) and PIK3C3/VPS34 (Martens et al., 2013, Zhou et al., 2010). Consistent with an important lens role for these genes, deletion of either gene results in retarded lens growth and in cataract formation demonstrating their importance for lens development, function and/or both.

Previous work showed that the human lens expresses the full complement of genes required to carry out autophagy (Brennan et al., 2012) and that these genes are expressed in both adult human lens epithelial cells and differentiating fiber cells. It has also been shown that autophagy can be induced in cultured lens epithelial cells demonstrating that autophagy could be a specific response of the lens exogenous changes (Brennan et al., 2012). Finally, it has been demonstrated that mutations in the autophagy gene FYVE and coiled coil domain containing 1 (FYCO1) cause autosomal recessive congenital human cataract providing further evidence that autophagy is essential for human lens development, transparency or both (Chen et al., 2011).

Here, we analyzed the presence of autophagolysosomes in human and embryonic chick lens epithelial cells and maturing lens fiber cells. We specifically focused on identifying mitochondria in autophagolysosomes since they are readily distinguishable by electron microscopy and by immune-specific confocal localization with recognizable mitochondrial and autophagosomal markers. Our analysis has identified the presence of large numbers of autophagolysosomes containing mitochondria and other material throughout the adult human and embryonic chick lens epithelial and fiber cells. We also demonstrate that serumstarvation, a standard method for inducing autophagy in multiple cell types (Klionsky et al., 2008) also induces mitophagy in primary chick lens epithelial cells suggesting that exogenous changes can induce mitophagy in lens cells Collectively, these data provide evidence that autophagy and mitophagy are significant features of the embryonic and adult lens that likely participate in the maintenance of lens cell homeostasis and the degradation of mitochondria and other organelles that occurs during lens fiber cell maturation. 


\section{Materials and Methods}

\subsection{Lenses}

Human transparent donor lenses from NC Eye Bank, Winston-Salem, NC, and Ramayamma International Eye Bank, Hyderabad, India, were obtained following the tenets of the Declaration of Helsinki for the protection of human subjects. Thirty lenses were processed (ages 22-92) and lenses of ages 22, 55 and 92 were examined in detail. Lenses were dissected and fixed immediately from day 12 chick embryos.

\subsection{Thin section electron microscopy}

The Vibratome method of ultrastructural analysis described previously (Costello et al., 2008) was employed with modifications to the initial fixation procedure. Briefly, human donor and embryonic chick lenses were fixed in $10 \%$ formalin for $24 \mathrm{~h}$ followed by fixation in freshly prepared $4 \%$ paraformaldehyde in $0.1 \mathrm{M}$ cacodylate buffer (Electron Microscopy Sciences (EMS), 12300) for $48 \mathrm{~h}$ (Costello et al., 2012). Fixed lenses were stored in $0.1 \mathrm{M}$ cacodylate buffer until Vibratome sectioning (Leica, model VT1000) of $200 \mu \mathrm{m}$ thick slices that were immersion fixed in $2.5 \%$ glutaraldehyde, $2 \%$ paraformaldehyde and $1 \%$ tannic acid in $0.1 \mathrm{M}$ cacodylate buffer ( $\mathrm{pH}$ 7.2). Sections were en bloc stained cold in $0.5 \%$ osmium tetroxide (EMS, 19100) for $60 \mathrm{~min}$, washed with deionized distilled water for three 15 min washings, washed once with $50 \%$ ethanol for $5 \mathrm{~min}$, stained in $2 \%$ uranyl acetate (ethanol-based; EMS, 22400) in the dark for $30 \mathrm{~min}$ and dehydrated through a graded ethanol series. Samples were embedded in an epoxy resin (EMS, Epon 812, 14120) and 70 $\mathrm{nm}$ thin sections were cut with a diamond knife (EMS, Diatome model Ultra45) from mesas raised to include the epithelium and outer cortex near the equatorial plane. Thin sections were grid stained with uranyl acetate and lead citrate for viewing at $80 \mathrm{kV}$ on a FEI Tecnai G2 transmission electron microscope (FEI, model T12) equipped with a high resolution slow scan CCD camera (Gatan, model 794) and digital montage software for imaging large areas.

The distribution of autophagolysosomes as a function of depth within the lens was measured in unit areas $\left(140 \mu^{2}\right)$ approximately equivalent to the cross-sectional area of seven fiber cells within the outer cortex. The unit areas were randomly selected along radial axes starting just beneath the epithelium. For human and chick lens sections, about ten areas to depths of $120 \mu \mathrm{m}$ and $250 \mu \mathrm{m}$, respectively, were recorded. All organelles were classified including vacuoles where the outer membrane was not clear and the contents were not present. Nuclei were present in some cells as expected, because the bow regions within the equatorial plane were examined. Mitochondria were identified by their double membranes, internal cristae and diameters near to $0.2 \mu \mathrm{m}$. Autophagolysosomes were present in all areas as vesicles of variable size bound with single membranes and containing heterogeneous contents.

\subsection{Co-Localization of LC3B and TOM20 in E12 chick lens sections}

Freshly isolated lenses were fixed in $3.7 \%$ formaldehyde overnight at $4{ }^{\circ} \mathrm{C}$ and transferred to $30 \%$ sucrose solution for cryopreservation. Lenses were prepared for cryosectioning and 20 $\mu \mathrm{m}$ thick sections were cut serially in the anterior to posterior direction. Mid-sagittal lens sections were then permeabilized with $0.25 \%$ Triton buffer for 10 mins, blocked in blocking buffer for $1 \mathrm{hr}$ (5\% goat serum, $0.5 \mathrm{gms}$ BSA in $50 \mathrm{mls}$ PBS) and then incubated sequentially in primary antibody overnight at $4^{\circ} \mathrm{C}$ followed by a fluorescent-conjugated secondary antibody (Jackson ImmunoResearch Laboratories, West Grove, PA) for $2 \mathrm{hrs}$ at $37^{\circ} \mathrm{C}$. Nuclei were coun terstained with TO-PRO-3. Image analysis was performed using the Zeiss LSM510 META confocal microscope. Single optical planes were selected from z-stacks, each $0.5 \mu \mathrm{m}$ thick using the LSM5 Image Browser. As control, no staining was observed using seconday antibody alone. 


\subsection{Preparation and treatment of chick primary lens epithelial cells}

Primary chick lens cell cultures were prepared from the lenses of White Leghorn embryonated chicken eggs (Charles River Laboratories) using the method of Menko et al. 1984. Briefly, primary lens cells were isolated from embryonic (E) 10 chick lenses by trypsinization and agitation. Cells were plated onto mouse laminin (Invitrogen, 23017015) and cultured in Medium 199 (11150067, Invitrogen) with 10\% FBS (Invitrogen, 10437028). For serum starvation cells were transferred into serum free M199 media for $2 \mathrm{~h}$.

\subsection{Co-Localization of LC3B and TOM20 in primary chick lens cells}

Primary chick lens epithelial cells were prepared as described above and plated onto laminin coated glass bottom 12 well plates. Cells were serum staved for $2 \mathrm{~h}$, washed in PBS and double immunofluoresence staining carried out. For co-localization, cells were fixed with $3.7 \%$ formaldehyde in PBS, permeabilized with $0.25 \%$ Triton X-100 in PBS and blocked with $1 \%$ BSA. Following blocking cells were washed with PBS and incubated overnight at 4 ${ }^{\circ} \mathrm{C}$ with primary antibodies . Primary antibodies were used at the following concentrations, LC3B (Sigma-Aldrich, L7543) at 1:500 and TOM20 (Santa Cruz, sc-17764) at 1:50. Following primary antibody incubation, cells were washed with 3 X PBS, and incubated with Alexa Fluor 488 goat anti-mouse secondary (Invitrogen, A31619) and/or Texas red goat anti-rabbit secondary (Invitrogen, T2767) for $1 \mathrm{~h}$ at room temperature, both at 1:2000 dilution. Cells were washed three times with PBS, and ProLong Gold Antifade mounting media added to the wells (Invitrogen, P36930). Immunofluoresent staining was visualized with a Zeiss LSM 700 Confocal microscope (Carl Zeiss)

\section{Results}

\subsection{Ultrastructural evidence for autophagy and mitophagy in the adult human lens epithelium}

The lens consists of a monolayer of epithelial cells on the anterior surface that overlies differentiating fiber cells. A prominent feature of the mammalian lens epithelium is the row of nuclei within cells having a constant cellular height; this is readily visualized here at low magnification of a male 22 year-old normal human donor lens (Fig. 1A). Higher magnification reveals the structure of the nuclear envelope and nuclear pores demonstrating that the lens cell structures and membranes are well preserved in this image (Fig. 1B). Within the cytoplasm, membranous organelles are clearly visible, including the endoplasmic reticulum and mitochondria $(\mathrm{m})$. Regions of cell debris and membrane aggregates within membranous vesicles are also present that are not typical of intact normal mitochondria and other organelles (Fig. 1B, arrowheads) and are thus potential examples of autophagic vesicles. Mitochondria are especially important because their distinctive morphology assists in evaluating their possible encapsulation and degradation within these structures by the autophagic machinery. Fortuitously, an intact rod-like mitochondrion is adjacent to an autophagic vesicle in the highlighted region (Fig. 1B), which is enlarged (Fig. 1C). The mitochondrion (Fig. 1C, m) is small (about $0.2 \times 1 \mu \mathrm{m}$ ), as is typical for the lens (Cohen, 1965), and has a clearly defined outer membrane and a complex inner membrane with numerous cristae. The adjacent autophagic vesicle has three fragments of mitochondria (Fig. $1 \mathrm{C}$, arrows) as well as other irregular cell components including a dark band which is a cluster of closely packed membranes. Other examples of autophagic vesicles containing mitochondria in different stages of degradation are illustrated (Fig. 1D, E). Components of mitochondria were identified using the criteria of size, roughly $0.2 \mu \mathrm{m}$ diameter, $1 \mu \mathrm{m}$ length, and appearance of outer membrane and inner membrane with cristae membrane fragments. These images demonstrate that the degrading mitochondrion is globular with cristae membranes preserved (Fig. 1D, m) or cylindrical with the cristae membrane not visible (Fig. 1E, m). Adjacent to the cylindrical degrading mitochondrion is a typical cluster 
of multilamellar membranes (Fig. 1E) with a lamellar repeat of about $5 \mathrm{~nm}$. This feature is normal of pure lipid bilayers without incorporated integral proteins (Gilliland et al, 2001) and is commonly observed in autophagic vesicles. The examples of autophagic vesicles in Fig. 1C-E are surrounded by a single membrane suggesting that they have fused with a lysosome and hydrolytic enzyme degradation has begun, so these should be termed autophagolysosomes. Because some of these autophagic vesicles contain mitochondria, there is clear evidence for both autophagy and mitophagy in this epithelium from a young adult human donor lens (22 y.o.). Similar examples of autophagy and mitophagy were also observed in epithelia from older human donor lenses (55 y.o. and 92 y.o.; data not shown) supporting the conclusion that autophagy and mitophagy mechanisms operate in human lens epithelium.

\subsection{Examples of autophagy and mitophagy are common features of the adult human lens differentiating fiber cells}

The presence of autophagic vesicles has not been previously demonstrated within the early differentiating lens fiber cells located in a region of the lens commonly called the lens cortex. In the same 22 year-old human donor lens, just beneath the lens epithelium in the equatorial plane of the lens, differentiating fiber cells in this so called bow region of the lens were examined in detail (Fig. 2A). An overview shows that potential autophagic vesicles are detected in the lens epithelium, elongating and differentiating fibers and early differentiated fibers (Fig. 2A, arrows). Only a few of the numerous cell degradation vacuoles present in this image are marked and each must be examined at high magnification to identify the characteristics of an autophagic vesicle including the outer limiting membranes and recognizable degrading heterogeneous cellular components. An ideal example (Fig. 2B) shows a single outer membrane (Fig. 2B, arrowhead) and cytoplasmic debris associated with a wavy membrane (left) and vesicular membranes (right) with large regions of multilamellar membranes (dark staining). It is important that these vesicles also display open spaces containing little or no stain, as these features are consistent with enzymatic degradation and identification of the vesicles at low magnification. An autophagolysosomes dominated by a multilamellar aggregate also contains a mitochondrial fragment (Fig. 2C, arrow).

Membranes from the multilamellar structure seem to blend with the outer limiting membrane, suggesting that lipid-rich membrane components may be recycled through redistribution into other intact membranes. In another autophagolysosome, a degrading mitochondrion is located by its cylindrical outline (Fig. 2D, arrow) adjacent to a multilamellar aggregate that appears to be connected to another mitochondrial fragment (Fig. 2D, arrowhead). In the center of this example sequestered protein is similar to the adjacent cytoplasmic protein. In two other examples, the sequestered protein is quite different (Figs. 2E and 2F). The core of protein in both examples appears more condensed than the adjacent cytoplasm and is associated with a band of multilamellar membranes (Fig. $2 \mathrm{E}$ ) or covered by several layers of loosely packed membranes (Fig. 2F). In the latter example taken from a deeper region of the cortex $(120 \mu \mathrm{m}$ from the lens capsule) the cytoplasm of the surrounding cells is condensed, signaling the initiation of the refractive index gradient of the lens. The core of the particle is even more condensed than the cytoplasm. Both examples (Figs. 2E and 2F) are very similar to the multilamellar bodies described within human nuclear cataracts (Gilliland et al., 2001; Gilliland et al., 2004; Gilliland et al., 2008).

The distribution of autophagic vesicles was estimated as a function of the distance from the epithelium-fiber cell interface in unit areas about the size of seven fiber cells. The number per unit area decreased linearly from about 16 to less than 5 within the first $60 \mu \mathrm{m}$ and remained low to greater than $120 \mu \mathrm{m}$ from the epithelium. Nuclei were present throughout 
this bow region indicating that the decrease in autophagic vesicles occurred outside of the OFZ. Intact mitochondria were visible throughout this region.

\subsection{Autophagy and mitophagy structures are present in the embryonic chick lens}

In order to explore the role of autophagy in the epithelium and young differentiating lens fiber cells, embryonic day 12 (E12) chick lenses were chosen as a model system. E12 lenses have not yet lost their mitochondria and other organelles to form the OFZ zone of the lens. An overview electron micrograph shows that the capsule near the equator and the annular pad was constant in thickness and slightly wavy, perhaps due to loss of elastic constraints during isolation (Fig. 3A). The epithelium displayed a complex pattern of cells with multiple layers and numerous vacuoles near the capsule, as has been reported previously (Shinohara et al., 1978). Within the cytoplasm of the epithelium and adjacent elongating fiber cells are numerous small regions of cell debris (Fig. 3A, arrows), which represent possible autophagic vesicles, as well as larger circular vacuoles of unknown origin (Fig. 3A, arrowhead). This pattern of focal cell disruption is continued into the young differentiating fiber cells just beneath the epithelium (Fig. 3B). A large number of structures that are likely autophagic vesicles are observed within the first ten cell layers (about $20 \mu \mathrm{m}$ from the epithelium; Fig. 3B, arrows). Deeper in the cortex, about $160 \mu \mathrm{m}$ from the epithelium, the cell shape remains uniformly hexagonal, although the number of potential autophagic vesicles is greatly diminished (Fig. 3C). Autophagic vesicles decreased linearly from about 25 per unit area (about seven fiber cells) to less than 5 within $250 \mu \mathrm{m}$ from the epithelium. This data suggests a pronounced gradient in autophagy within the cortex leading away from the epithelium. Identification of the type of autophagy depends on examination of high magnification images. In the epithelium, intact mitochondria can be found near to endoplasmic reticulum (Fig. 3D). An example of mitophagy can be found within the epithelial cytoplasm where the degrading mitochondrion is adjacent to a multilamellar aggregate (Fig. 3E). Four examples of mitophagy are illustrated from the region of fiber cells just below the epithelium (Figs. 3F-I). In each case membranes most likely derived from cristae are located (Figs. 3F-I, arrows) to confirm the identification of the degrading organelle. Multilamellar aggregates are present in three of the examples in different configurations (Figs. 3F, 3G and 3I). Fiber cells deeper into the cortex also show similar examples of autophagy, such as the autophagolysosomes located about $160 \mu \mathrm{m}$ from the epithelium (Fig. 3C, arrow) shown at high magnification (Fig. 3J). The single outer limiting membrane surrounds a large central region of degrading cytoplasmic protein and peripheral regions contain multilamellar membranes and degrading organelles. In the same region an autophagolysosomes with a dense core of cytoplasmic crystallins surrounded by multilamellar membranes can be found (Fig. 3K, arrow) which is very similar to multilamellar bodies in human nuclear cataracts (Gilliland et al., 2001; Gilliland et al., 2004; Gilliland et al., 2008). These images provide strong evidence for the existence of autophagy and mitophagy in both epithelial and fiber cells of the developing chick lens outside the OFZ zone at a stage where all cells still contain nuclei (Bassnett, 2009). Furthermore, the gradient of autophagy within the cortex suggests that autophagy is likely to contribute to membranous organelle degradation during lens cell differentiation.

\subsection{Co-Localization of the autophagy marker LC3B with the mitochondrial marker TOM20 in the embryonic chick lens}

To further confirm the presence of autophagy and mitophagy in the lens and to spatially identify regions of active autophagy and mitophagy in the developing chick lens, we colocalized the specific autophagosomal marker LC $3 \mathrm{~B}$ and the mitochondrial marker translocase of the outer mitochondrial membrane $20 \mathrm{kDa}$ (TOM20) on midsagittal lens sections from E12 chicken lenses. Low magnification imaging of TOM20 (green) and LC3B (red) indicate that co-localization of these markers occurs in specific areas of the developing 
chick lens (Fig. 4). Examination of the cortical fiber cells zone (FP) of the embryonic lens (Fig. 4B), where immature lens fibers cells undergo a dramatic elongation process, reveals a number of LC3B positive (red) puncta that co-localized with TOM20-labelled (green) mitochondria. Yellow puncta indicate a region of LC3B/TOM20 overlap. In this area of the lens, differentiating lens fiber cells are beginning to lose their mitochondria and the colocalization of the mitochondria marker TOM20 with LC3B suggests that degradation of these mitochondria is mediated, at least in part, by autophagy and mitophagy mechanisms. Co-localization of LC3B and TOM20 (yellow puncta) occurred even more frequently in the maturing lens cells in the central fiber zone (FC). The lens equatorial zone where lens epithelial cells initiate their differentiation (EQ, Fig. 4D) revealed that autophagic vesicles are present in the epithelium prior to fiber cell differentiation. The extensive distribution of mitochondria as shown by staining with TOM20 (green), make it difficult to determine whether there are mitochondria that are being degraded in autophagic vesicles in this region. However, in the most peripheral cortical fiber cells some puncta that were co-labeled with TOM20 and LC3B (yellow) could be detected (arrows), as well as LC3B-positive autophagic vesicles that do not contain fragments of mitochondria. Collectively, these data indicate the presence of mitochondria within autophagosomal components throughout the spectrum of fiber cell differentiation in the 12 day chick lens provide evidence that mitophagy is a significant feature of lens cell maturation.

\subsection{Co-localization of LC3B and the mitochondrial marker TOM20 in serum starved chick primary lens cells}

Serum starvation has been demonstrated to induce autophagy and mitophagy in multiple cell-types (Ravikumar et al., 2009) so we used it here to examine if lens cells could respond to environmental changes through induction of autophagy as evidenced in our previous study (Brennan et al., 2012). This also demonstrates that all the necessary macroautophagy machinery is present and functional in lens cells. To determine if autophagy/mitophagy is a response of lens cells to serum starvation, chick lens primary epithelial cells were exposed to serum starvation for 2 hours and labeled for markers of autophagy induction. These studies were performed in the presence and absence of $50 \mu \mathrm{M}$ chloroquine. Chloroquine prevents autophagosomal fusion with lysosomes allowing visualization of accumulated LC3B II stained autophagosomes that would otherwise be turned over and the signal lost. We observed increased co-localization of LC3B-positive puncta (red) with TOM20 positive mitochondria (green) in serum-starved chick primary lens epithelial cells exposed to $50 \mu \mathrm{M}$ chloroquine for $2 \mathrm{~h}$ relative to cells maintained in complete media and exposed to $50 \mu \mathrm{M}$ chloroquine (Fig. 5). Co-localization of the LC3B (red) and TOM20 (green) signals revealed little co-localization of TOM20 with LC3B in the control cells but large areas of colocalization (orange/yellow color) in the serum starved cells particularly in the perinuclear region of the cells, suggesting that autophagy-mediated mitochondrial degradation occurs upon serum starvation of these cells (Fig. 5). Co-localization was also examined in the absence of chloroquine and none was found (data not shown).

\section{Discussion}

The morphological and immunofluorecent confocal microscopy data presented here demonstrate the presence of autophagic vesicles in the ocular lens in lens epithelial cells and differentiating lens fiber cells. These structures were detected in large numbers in both adult human and embryonic chick lenses. These structures contained both cytoplasmic material and mitochondria in different states of degradation. Since these structures arise as a consequence of autophagosome fusion with lysosomes these data provide evidence that both autophagy and mitophagy participate in the degradation of mitochondria and other cellular organelles and material in lens epithelial and fiber cells. These structures also contained 
significant levels of multilamellar lipid aggregates suggestive of cell membrane degradation that could be used for cellular recycling. Finally, we demonstrate that serum-starvation results in increased co-localization of mitochondrial and autophagy markers in embryonic chick lens cells suggesting that mitophagy in lens cells can be induced in response to extracellular conditions that induce mitophagy in other cells (Klionsky et al., 2008).

The data demonstrate that autophagy and mitophagy are processes in lens cells that are likely are important for the maintenance of lens homeostasis through the degradation of damaged mitochondria (Youle and Narendra 2011) and in the clearance of mitochondria during lens fiber cell maturation, contributing to the formation of the OFZ of the lens. The lens epithelium, is required for the maintenance of the entire lens through ionic transporters that maintain correct ion and metabolite concentrations in the lens fibers (Goodenough, 1992). Disruption of these systems results in loss of lens homeostasis and cataract formation, since they all require mitochondrial energy in the form of ATP and reducing equivalents (Brennan and Kantorow, 2009). Thus, failure to degrade damaged mitochondria in the lens epithelium by mitophagy would result in loss of the energy required for the function of essential lens enzyme and transport systems, with concomitant increased reactive oxygen species production, oxidation and ultimately loss of lens homeostasis and cataract formation. The newly formed fiber cells of the lens require mitochondrial energy for crystallin synthesis, cellular elongation and cytoskeletal remodeling (Bassnett, 2011). Accumulation of dysfunctional mitochondria in the absence of mitophagy would therefore disrupt lens fiber protein synthesis and fiber cell differentiation leading to loss of lens development and dysfunctional lens formation. Lastly, mitochondria are eliminated during lens fiber cell maturation to form the OFZ of the lens, to date the mechanism responsible for this elimination is unknown (Bassnett, 2009). The data presented here showing mitochondria in autophagosomes throughout adult human and developing chick lenses, suggests that mitophagy is at least one mechanism by which mitochondria are cleared during fiber cell maturation. Therefore, loss of mitophagy would result in retention of mitochondria in this lens region, leading to light scattering and cataract formation.

Previous reports have provided conflicting evidence regarding the roles of autophagy and mitophagy in lens organelle degradation. Most recently, a study of targeted-knockout of the autophagy genes Atg5 and Pik3c3 in the mouse lens demonstrated that deletion of either of these genes resulted in retarded lens growth, grossly altered lens morphology and cataract formation (Morishita et al., 2013). These data support the assertion that autophagy genes are indeed essential for lens development, however they do not clarify the potential role of autophagy or mitophagy in removing mitochondria and other organelles during lens fiber cell maturation since neither of these pathways is an absolute requirement for autophagosome formation (Nishida et al., 2009, recent Autophagy paper Martens et al. 2013, Zhou et al. 2011).

The ultrastructural data presented here support the hypothesis that autophagy produces the precursors to multilamellar bodies in the lens that have been previously described as 1-4 $\mu \mathrm{m}$ diameter spherical particles with a protein core and multiple layers of bilayer lipid coat (Gilliland et al., 2001; Gilliland et al., 2004). Although multilamellar bodies have been described in many organs and cell types (Gilliland et al., 2001), including cortical cataracts from aging rats (Gorthy, 1978), the term is used here to identify particles that are likely sources of scattering in human transparent lenses and age-related nuclear cataracts (Costello et al., 2007; 2010). It was hypothesized previously that multilamellar bodies may be autophagosomes that were not completely cleared (Gilliland et al., 2004) and this idea is supported by the remarkable similarity to multilamellar bodies of some of the autophagic vesicles reported here in Figs 2E, $2 \mathrm{~F}$ and $3 \mathrm{~K}$. The observation of nearly spherical dense cores of cytoplasmic proteins in autophagic vesicles may be unique to the lens suggesting 
that the lens may have a mechanism to identify regions of cytoplasm for degradation even after the proteins begin to condense. Such unusual morphology supports the speculation that the particles may resist degradation and result in the multilamellar bodies in adult lenses. It should be noted that the reported location of multilamellar bodies in adult human lenses and nuclear cataracts within the fetal and embryonic nuclei is consistent with fluorescent autophagic particles in a transgenic mouse model (Matsui et al., 2006) and in the E12 embryonic chick lenses reported here (Fig. 4).

In summary, the present data provide evidence that autophagy and mitophagy operate at significant levels in the lens where they likely function to degrade and recycle damaged mitochondria and other organelles throughout the entire lens and in the possible elimination of mitochondria and other organelles during lens fiber cell maturation. Collectively these data suggest that loss of these mechanisms could result in altered lens development, loss of lens homeostasis and cataract formation.

\section{Acknowledgments}

This work was supported by NIH/NEI RO1 grants EY008148 (MJC) and EY13022 (MK) and EY010577 (SM). We thank Mr. Hal Mekeel for his expert technical assistance. We appreciate the assistance of Dr. W. Craig Fowler in obtaining donor lenses from the NC Eye Bank.

\section{Abbreviations}

$\begin{array}{ll}\text { OFZ } & \text { Organelle free zone } \\ \text { LC3B } & \text { microtubule-associated protein 1 light chain 3B } \\ \text { FYCO1 } & \text { FYVE and coiled coil domain containing 1 } \\ \text { TOM20 } & \text { translocase of the outer mitochondrial membranes 20 kDa } \\ \text { ATG5 } & \text { autophagy-related 5 } \\ \text { Pik3c3 } & \text { Phosphatidylinositol 3-Kinase, Catalytic Subunit Type 3 }\end{array}$

\section{References}

Bassnett S. Mitochondrial dynamics in differentiating fiber cells of the mammalian lens. Curr. Eye Res. 1992; 11:1227-32. [PubMed: 1490341]

Bassnett $\mathrm{S}$. The fate of the Golgi apparatus and the endoplasmic reticulum during lens fiber cell differentiation. Invest Ophthalmol Vis Sci. 1995; 36:1793-803. [PubMed: 7635654]

Bassnett S. On the mechanism of organelle degradation in the vertebrate lens. Exp Eye Res. 2009; 88:133-39. [PubMed: 18840431]

Bassnett S, Shi Y, Vrensen GF. Biological glass: structural determinants of eye lens transparency. Philos Trans R Soc Lond B Biol Sci. 2011; 366:1250-64. [PubMed: 21402584]

Bloemendal, H., editor. Molecular and cellular biology of the eye lens. Wiley; New York: 1981.

Brennan LA, Kantorow M. Mitochondrial function and redox control in the aging eye: role of MsrA and other repair systems in cataract and macular degenerations. Exp Eye Res. 2009; 88:195-203. [PubMed: 18588875]

Brennan LA, Lee W, Cowell T, Giblin F, Kantorow M. Deletion of mouse MsrA results in HBOinduced cataract: MsrA repairs mitochondrial cytochrome c. Mol Vis. 2009; 15:985-99. [PubMed: 19461988]

Brennan LA, Kantorow WL, Chauss D, McGreal RS, He S, Mattucci L, Wei J, Riazuddin SA, Cvekl A, Hejtmancik JF, Kantorow M. Spatial expression patterns of autophagy genes in the eye lens and induction of autophagy in lens cells. Mol Vis. 2012; 18:1773-86. [PubMed: 22815631]

Exp Eye Res. Author manuscript; available in PMC 2014 November 01. 
Brennan LA, McGreal RS, Kantorow M. Oxidative stress defense and repair systems of the ocular lens. Front Biosci. (Elite Ed). 2012; 4:141-55. [PubMed: 22201860]

Brown, NP.; Bron, NJ., editors. Lens Disorders: A Clinical Manual of Cataract Diagnosis. Butterworth-Heinemann; Oxford: 1996.

Chen J, Ma Z, Jiao X, Fariss R, Kantorow WL, Kantorow M, Pras E, Frydman M, Pras E, Riazuddin S, Riazuddin SA, Hejtmancik JF. Mutations in FYCO1 cause autosomal-recessive congenital cataracts. Am J Hum Genet. 2011; 88:827-38. [PubMed: 21636066]

Cohen AI. The electron microscopy of the normal human lens. Invest Ophthalmol. 1965; 4:433-46. [PubMed: 14340160]

Costello MJ, Johnsen S, Gilliland KO, Freel CD, Fowler WC. Predicted Light Scattering from Particles Observed in Human Age-Related Nuclear Cataracts Using Mie Scattering Theory. Invest Ophthalmol Vis Sci. 2007; 48:303-312. [PubMed: 17197547]

Costello MJ, Gilliland KO, Metlapally S, Ramamurthy B, Krishna PV, Balasubramanian D. Ultrastructural analysis of damage to nuclear fiber cell membranes in advanced cataracts from India. Exp Eye Res. 2008; 87:147-158. [PubMed: 18617164]

Costello MJ, Johnsen S, Metlapally S, Gilliland KO, Frame L, Balasubramanian D. Multilamellar spherical particles as potential sources of excessive light scattering in human age-related nuclear cataracts. Exp Eye Res. 2010; 91(6):881-9. [PubMed: 20888812]

Costello MJ, Mohamed A, Gilliland KO, Metlapally S, Johnsen S, Fowler WC. Simple fixation and storage protocol for preserving the internal structure of intact donor lenses and extracted human nuclear cataracts. Invest Ophthalmol Vis Sci. 2012; 52 ARVO eAbstract 3031.

Delamere NA, Tamiya S. Lens ion transport: from basic concepts to regulation of Na,K-ATPase activity. Exp. Eye Res. 2009; 88:140-143. [PubMed: 18614168]

De Maria A, Bassnett S. DNase IIbeta distribution and activity in the mouse lens. Invest Ophthalmol Vis Sci. 2007; 48:5638-46. [PubMed: 18055814]

Girão H, Pereira P, Taylor A, Shang F. Subcellular redistribution of components of the ubiquitinproteasome pathway during lens differentiation and maturation. Invest Ophthalmol Vis Sci. 2005; 46:1386-92. [PubMed: 15790906]

Gilliland KO, Freel CD, Lane CW, Fowler WC, Costello MJ. Multilamellar bodies as potential scattering particles in human age-related nuclear cataracts. Mol Vis. 2001; 7:120-30. [PubMed: 11435998]

Gilliland KO, Freel CD, Johnsen S, Fowler WC, Costello MJ. Distribution, spherical structure and predicted Mie scattering of multilamellar bodies in human age-related nuclear cataracts. Exp Eye Res. 2004; 79:563-76. [PubMed: 15381040]

Gilliland KO, Johnson S, Metlapally S, Costello MJ, Ramamurthy B, Krishna PV, Balasubramanian D. Mie light scattering calculations for multilamellar bodies in Indian age-related nuclear cataracts. Mol Vis. 2008; 14:572-82. [PubMed: 18385793]

Goodenough DA. The crystalline lens. A system networked by gap junctional intercellular communication. Semin Cell Biol. 1992; 3(1):49-58. [PubMed: 1320431]

Gorthy W. Cataracts in the aging rat lens. Morphology and acid phosphatase histochemistry of incipient forms. Exp Eye Res. 1978; 27:301-22. [PubMed: 710542]

Gronowicz G, Swift H, Steck TL. Maturation of the reticulocyte in vitro. J Cell Sci. 1984; 71:177-97. [PubMed: 6097593]

Itakura E, Kishi-Itakura C, Koyama-Honda I, Mizushima N. Structures containing Atg9A and the ULK1 complex independently target depolarized mitochondria at initial stages of Parkin-mediated mitophagy. J Cell Sci. 2012; 125:1488-99. [PubMed: 22275429]

Kantorow M, Lee W, Chauss D. Focus on Molecules: methionine sulfoxide reductase A. Exp Eye Res. 2012; 100:110-1. [PubMed: 20888813]

Klionsky DJ, Abeliovich H, Agostinis P, Agrawal DK, Aliev G, Askew DS, et al. Guidelines for the use and interpretation of assays for monitoring autophagy in higher eukaryotes. Autophagy. 2008; 4:151-75. [PubMed: 18188003]

Levine B, Klionsky DJ. Development by self-digestion: molecular mechanisms and biological functions of autophagy. Dev Cell. 2004; 6:463-77. [PubMed: 15068787]

Martens S, Rusten TE, Kraft C. Autophagy at sea. Autophagy. 2013; 9 epub ahead of print. 
Matsui M, Yamamoto A, Kuma A, Ohsumi Y, Mizushima N. Organelle degradation during the lens and erythroid differentiation is independent of autophagy. Biochem Biophys Res Commun. 2006; 339:485-9. [PubMed: 16300732]

Menko AS, Klukas KA, Johnson RG. Chicken embryo lens cultures mimic differentiation in the lens. Dev Biol. 1984; 103:129-41. [PubMed: 6370757]

Morishita H, Eguchi S, Kimua H, Sasaski J, Sakamaki Y, Robinson ML, Sasaki T, Mizushima N. Deletion of autophagy-related 5 (Atg5) and Pik3c3 in the lens causes cataract independent of programmed organelle degradation. J Biol. Chem. 2013; 288(16):11436-47. [PubMed: 23479732]

Mortensen M, Ferguson DJ, Simon AK. Mitochondrial clearance by autophagy in developing erythrocytes: clearly important, but just how much so? Cell Cycle. 2010; 9:1901-6. [PubMed: 20495377]

Nishida Y, Arakawa S, Fujitani K, Yamaguchi H, Mizuta T, Kanaseki T, Komatsu M, Otsu K, Tsujimoto Y, Shimizu S. Discovery of Atg5/Atg7-independent alternative macroautophagy. Nature. 2009; 461:654-8. [PubMed: 19794493]

Pendergrass WR, Penn PE, Possin DE, Wolf NS. Accumulation of DNA, nuclear and mitochondrial debris, and ROS at sites of age-related cortical cataract in mice. Invest Ophthalmol Vis Sci. 2005; 46:4661-70. [PubMed: 16303963]

Pendergrass W, Zitnik G, Urfer SR, Wolf N. Age-related retention of fiber cell nuclei and nuclear fragments in the lens cortices of multiple species. Mol Vis. 2011; 17:2672-84. [PubMed: 22065920]

Rabl C. Über den bau und die entwicklung der linse. III Teil: die lines der säugethiere. Ruckblick und schluss. Z. Wiss. Zool. 1899; 67:1-138.

Ravikumar B, Futter M, Jahreiss L, Korolchuk VI, Lichtenberg M, Luo S, Massey DC, Menzies FM, Narayanan U, Renna M, Jimenez-Sanchez M, Sarkar S, Underwood B, Winslow A, Rubinsztein DC. Mammalian macroautophagy at a glance. J Cell Sci. 2009; 122:1707-11. [PubMed: 19461070]

Shinohara T, Robison WG Jr, Piatigorsky J. Delta-crystallin synthesis and vacuole formation during induced opacification of cultured embryonic chick lenses. Invest Ophthalmol Vis Sci. 1978; 17:515-22. [PubMed: 659072]

Yang Z, Klionsky DJ. Mammalian autophagy: core molecular machinery and signaling regulation. Curr Opin Cell Biol. 2010a; 22:124-31. [PubMed: 20034776]

Yang Z, Klionsky DJ. Eaten alive: a history of macroautophagy. Nat Cell Biol. 2010b; 12:814-22. [PubMed: 20811353]

Youle RJ, Narendra DP. Mechanisms of mitophagy. Nat Rev Mol Cell Biol. 2011; 12:9-14. [PubMed: 21179058]

Youle RD, van der Bliek AM. Mitochondrial fission, fusion, and stress. Science. 2012; 337:1062-5. [PubMed: 22936770]

Zandy AJ, Bassnett S. Proteolytic Mechanisms Underlying Mitochondrial Degradation in the Ocular Lens. Invest Ophthalmol Vis Sci. 2007; 48:293-302. [PubMed: 17197546]

Zhou X, Wang L, Hasegawa H, Amin P, Han BX, Kaneko S, He Y, Wang F. Deletion of PIK3C3/ Vps34 in sensory neurons causes rapid neurodegeneration by disrupting the endosomal but not the autophagic pathway. Proc Natl Acad Sci U S A. 2010; 107:9424-9. [PubMed: 20439739]

Exp Eye Res. Author manuscript; available in PMC 2014 November 01. 


\section{Highlights}

- Organelle degradation is critical for lens homeostasis and lens cell maturation.

- Failure to degrade damaged organelles or organelles during lens cell maturation results in cataract formation.

A Autophagy is a novel mechanism for lens organelle degradation 

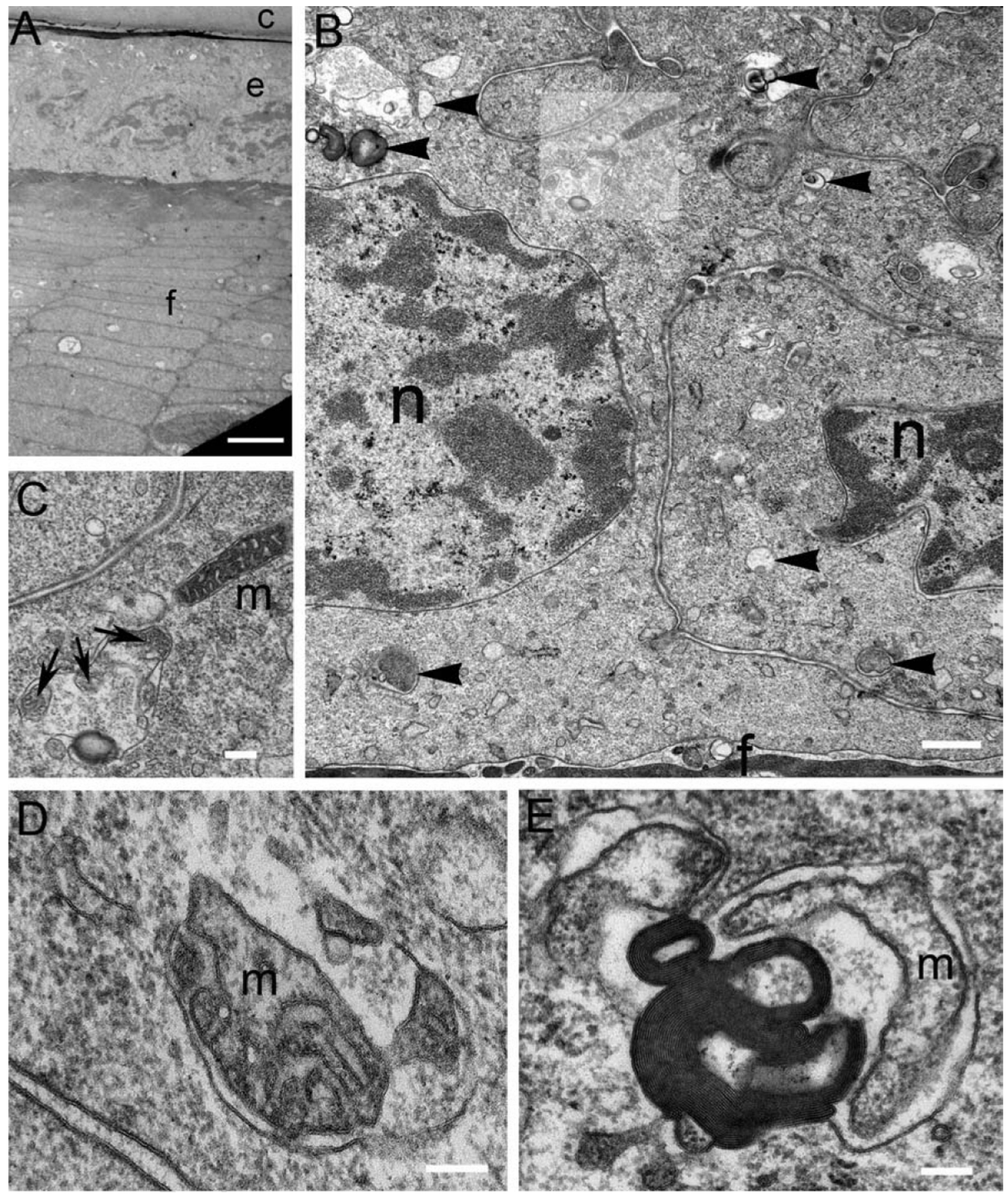

Figure 1. Autophagy and mitophagy in adult human lens epithelial cells

(A) Overview of lens surface from the 22 yo donor lens from India showing the capsule (c), epithelium (e) and fiber cells (f). (B) Two cells of the epithelium, separated by paired plasma membranes, display nuclei (n) and numerous autophagic vesicles within the epithelial cytoplasm (arrowheads). At the epithelial-fiber cell interface fiber cells (f) are stained darkly. The highlighted region is enlarged in (C). (C) An intact mitochondrion (m) is adjacent to an autophagic vesicle containing mitochondrial fragments (arrows). This is a clear example of mitophagy. (D) An autophagic vesical containing a partially degraded mitochondrion ( $\mathrm{m}$; based on the cylindrical profile about $0.2 \mu \mathrm{m} \times 0.7 \mu \mathrm{m}$ ). (E) An 
autophagic vesicle probably containing a mitochondrion (m) and a multilamellar membrane aggregate found in many autophagic vesicles ( $5 \mathrm{~nm}$ average membrane thickness). Scale bars: $\mathrm{A}=5 \mu \mathrm{m} ; \mathrm{B}=0.5 \mu \mathrm{m} ; \mathrm{C}=200 \mathrm{~nm} ; \mathrm{D}, \mathrm{E}=100 \mathrm{~nm}$. 

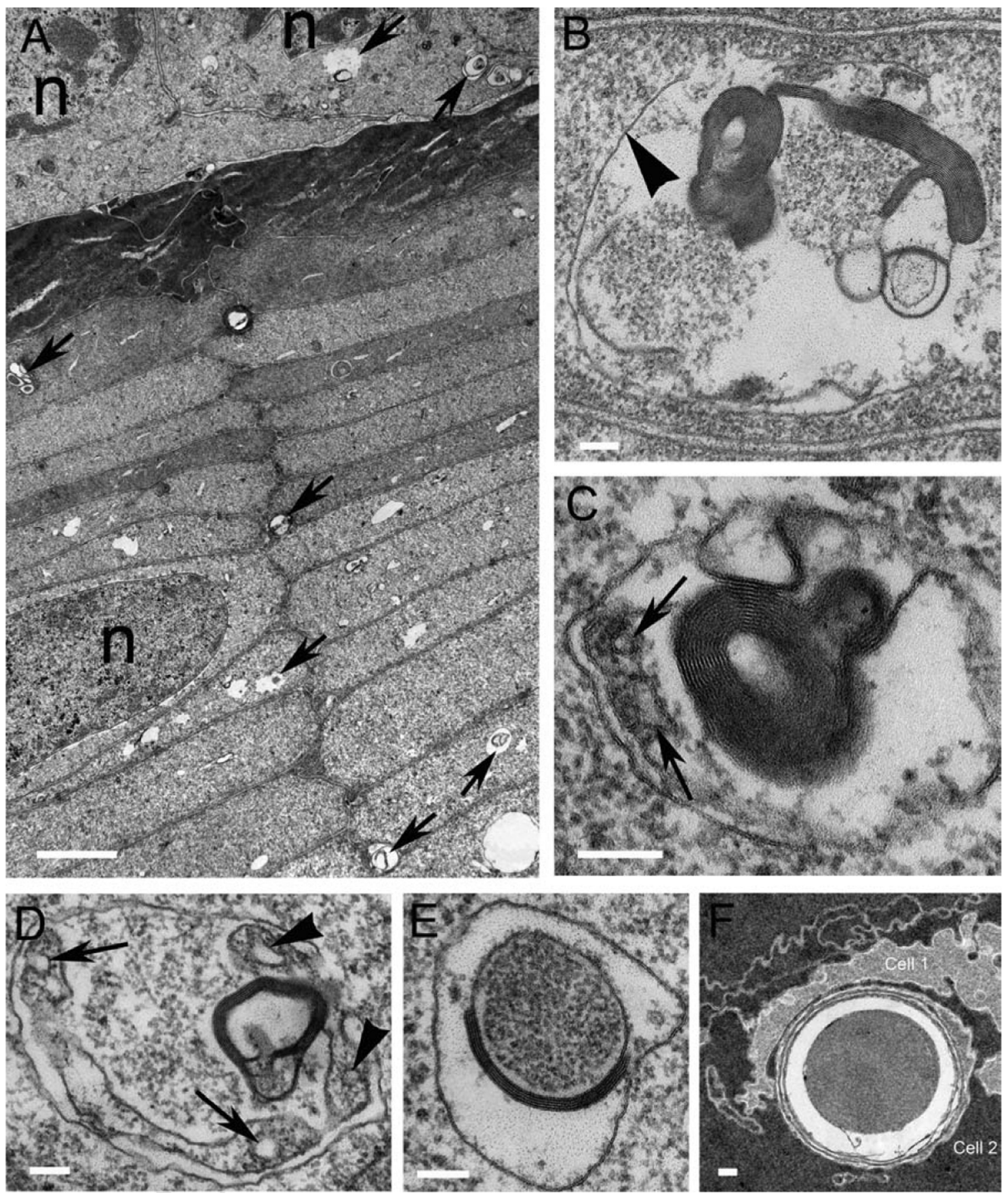

Figure 2. Autophagy and mitophagy in adult human lens cortical fiber cells

(A) Overview of the outer cortical fiber cells from the 22 yo donor lens from India. Two nuclei of the epithelium and one from a fiber cell are labeled (n). Two autophagic vesicles in the epithelium are marked and five from the fiber cells (arrows). Note the dark staining of the initial layers of elongating fiber cells and the relatively light staining of most of the fiber cells. The density of autophagic vesicles in this region, confirmed at high magnification, is about 16 per a unit area (of about seven fiber cells in cross-section) and drops to less than 5 by $60 \mu \mathrm{m}$ from the epithelium. (B) An autophagic vesicle between two adjacent fiber cells indicated by the paired membranes at the top and bottom of the image. The limiting membrane of the autophagic vesicle is indicated (arrowhead) and surrounds distinct 
components including multilamellar membranes (dark staining), vesicles and cell fragments composed of membranes and dispersed proteins. (C) The autophagic vesicle contains multilamellar membranes (dark staining) and a fragment of a mitochondrion (cristae are labeled, arrows). This is an example of mitophagy. (D) A large autophagic vesicle with properties similar to those in (B) and (C) containing a band of multilamellar membranes, dispersed protein in the center, an elongated mitochondrion (arrows) and a smaller mitochondrial fragment in contact with the multilamellar aggregate (cristae are labeled, arrowhead). (E) An autophagic vesicle with an oval core containing protein packed more densely than the adjacent cytoplasm and partially surrounded by tightly packed multiple membranes. (F) An autophagic vesicle near the interface between two fiber cells (Cells 1 and 2) containing a dense uniform core (more dense than the adjacent cytoplasm) and loosely packed multiple membranes. From a 92 yo donor lens from India at a location in the equatorial plane about $120 \mu \mathrm{m}$ from the capsule. Scale bars: $\mathrm{A}=2 \mu \mathrm{m} ; \mathrm{B}-\mathrm{E}=100 \mathrm{~nm} ; \mathrm{F}=$ $200 \mathrm{~nm}$.

Exp Eye Res. Author manuscript; available in PMC 2014 November 01. 

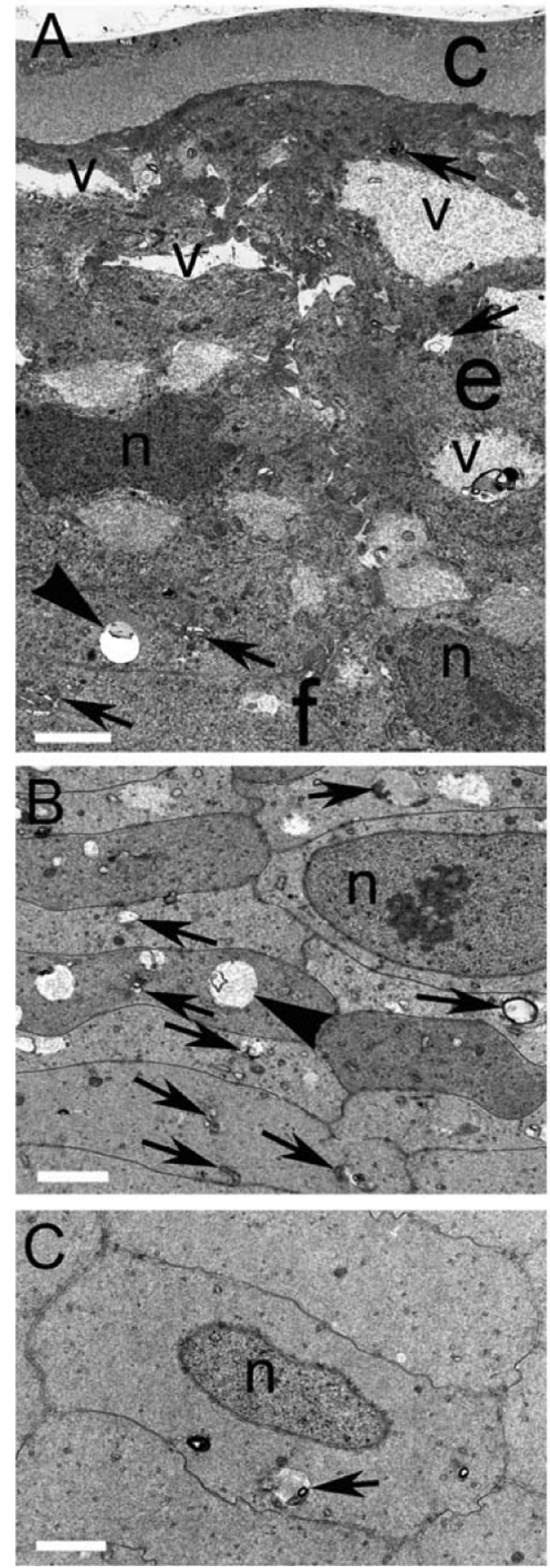
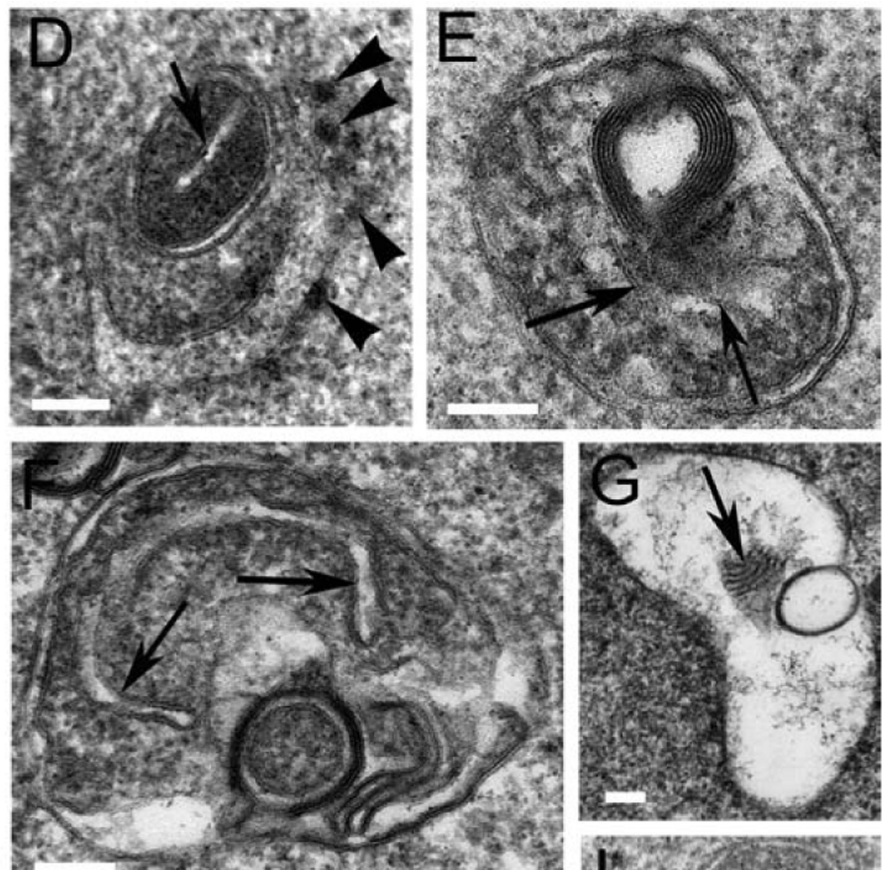

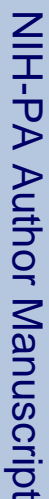

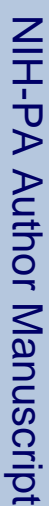

Figure 3. Autophagy and mitophagy in day 12 chick embryo lens

(A) Overview of capsule (c) and epithelium (e) of the embryonic chick lens. Two nuclei (n) are indicated from the epithelium, which has multiple layers and many vacuoles (v) typical of the complex avian developing epithelium. Numerous mitochondria and mitophagic vesicles (arrows) are present in the epithelium and in the early fiber cells (f). Large empty vesicles (arrowhead) are also common but may not be autophagic as their perimeter and contents cannot easily be identified. (B) Just beneath the epithelium are developing fiber cells with nuclei (n), large vesicles (arrowhead) and numerous autophagic particles (arrows), eight of which are indicated out of more than twenty visible at higher magnification. This region contains about 25 autophagic vesicles per unit area. (C) About $160 \mu \mathrm{m}$ deep in the 
cortex, the fiber cells are clearly defined from their hexagonal shape and nuclei (n). Very few autophagic vesicles (arrow) are present here indicating a pronounced gradient of autophagy through the cortex reducing to less than 5 per unit area by $250 \mu \mathrm{m}$ from the epithelium. (D) An intact mitochondrion (arrow) is adjacent to a portion of ER with a few ribosomes (arrowheads) from the epithelium (as in A). (E) An autophagic vesicle from the epithelium containing a mitochondrion (arrows) adjacent to multilayered membranes ( $5 \mathrm{~nm}$ average membrane thickness). (F) An autophagic vesicle containing a degrading mitochondrion having one clearly visible cristae (arrows). Images F-I are from young cortical fiber cells as in B where autophagy and mitophagy are very active. (G) An autophagic vesicle most likely containing a cluster of partially degraded mitochondrial cristae (arrow). (H) An autophagic vesicle similar to F except several smaller segments of cristae are visible (arrows). (I) An autophagic vesicle containing a degrading mitochondrion in the upper region with cristae (arrows) and the lower region contains degrading multilamellar membranes. (J) An autophagic vesicle adjacent to an intact mitochondrion (arrow) in the fiber cell displayed in C. (K) An autophagic vesicle containing a dense core of protein surrounded my multiple membrane layers (arrow) from the same region of fiber cells as in C. Scale bars: $A=2 \mu \mathrm{m} ; \mathrm{B}, \mathrm{C}=1 \mu \mathrm{m} ; \mathrm{D}-\mathrm{K}=100 \mathrm{~nm}$. 

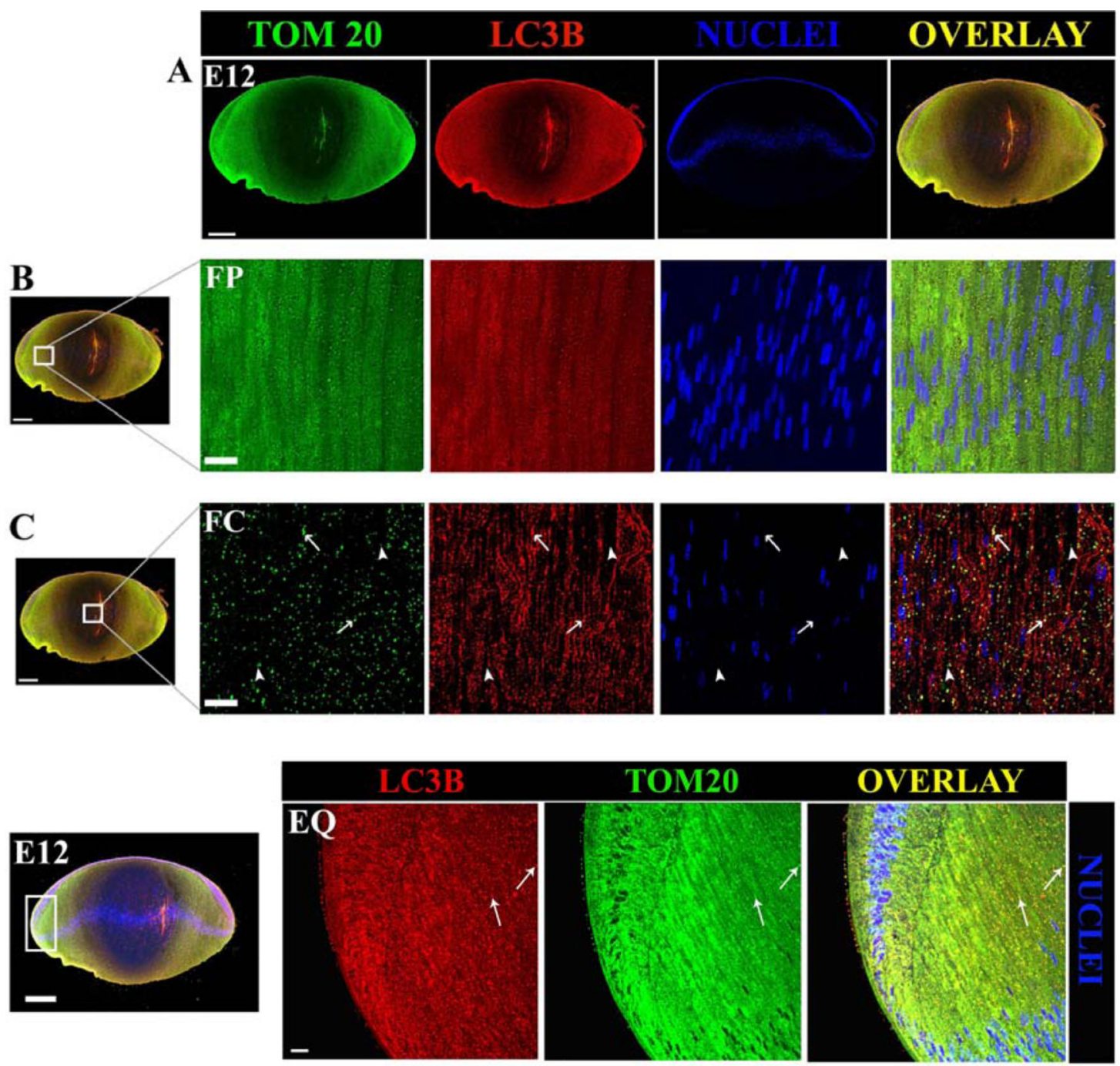

\section{TOM20}

OVERLAY

Figure 4. Co-Localization of the autophagy marker LC3B and the mitochondrial marker TOM20 in the E12 embryonic chick lens

Co-localization of TOM20 (green) and LC3B (red) puncta and overlay of the two images (orange/yellow) in E12 chick lenses using confocal fluorescent microscopy. (A) A $20 \mu \mathrm{m}$ midsagittal section from an E12 whole embryonic chicken lens stained for TOM20 (green), LC3B (red) and nuclear stain TO-PRO-3 (blue). (B) FP (cortical fiber) region of the chick lens and (C). FC (central fiber core) region of the chick lens. (D) EQ (equatorial region) of the chick lens. Fig. 4D is valuable for demonstrating (a) multiple layers of nuclei in the epithelium consistent with the TEM in Fig. 3, (b) the great amount of autophagic vesicles (LC3B dots) and mitochondria (TOM20 dots) in the equatorial region, (c) correspondence of the dots in the overlay suggesting many of the mitochondria are undergoing autophagy, and (d) there is more autophagy here than in the core (Fig. 4C) suggesting a radial decrease in autophagy from the epithelium to the core consistent with the gradient found in the TEM images (Fig. 3). Arrows indicate TOM20 green and LC3B red puncta and their colocalization (orange/yellow color in overlayed images) in each sub-compartment of the lens examined. 

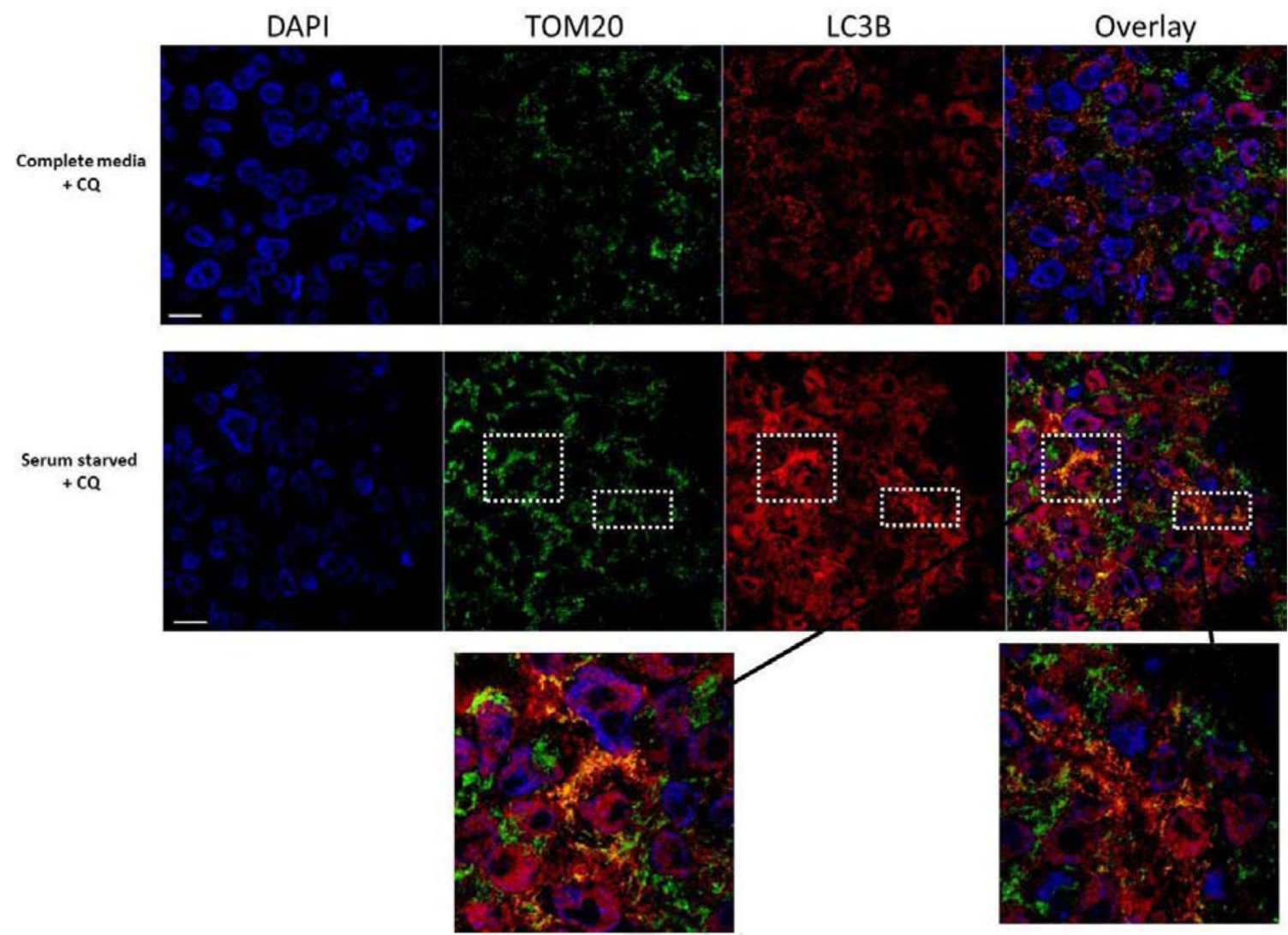

Figure 5. Co-Localization of the autophagy marker LC3B and the mitochondrial marker TOM20 in control and serum-starved chick lens epithelial cells treated with chloroquine Chick lens primary cells stained for TOM20 (green) and LC3B (red) in cells maintained in complete media and cells that were serum-starved for $2 \mathrm{~h}$ with the addition of $50 \mu \mathrm{M}$ chloroquine to both. Images were obtained using fluorescent confocal microscopy. Two areas with large numbers of perinuclear localized LC3B positive mitochondria (yellow puncta) are shown at higher magnification. 